\title{
Kyste De L’ouraque Infecte : A Propos De Trois Cas Cliniques Au Chu De Parakou Au Benin
}

\author{
Hodonou Montcho Adrien \\ Allodé Salako Alexandre \\ Tamou sambo Bio \\ Ossé Martin \\ Moumouni Amina \\ Mensah Emile
}

Département de chirurgie et spécialités chirurgicales, Faculté de Médecine,

Université de Parakou-Bénin, Centre Hospitalier Universitaire

Départemental du Borgou-Alibori

Mehinto Kouassi Delphin

Clinique universitaire de chirurgie viscérale B, Centre National Hospitalier et

Universitaire HKM Cotonou-Bénin

doi: 10.19044/esj.2016.v12n3p134 URL:http://dx.doi.org/10.19044/esj.2016.v12n3p134

\begin{abstract}
Bacground: The urachus is a fibrous remnant of the allantois. That connects the bladder dome to the anterior abdominal wall. After birth it is obliterated in general. In some cases it may persist as cyst, fistula , sinus or diverticulum. This persistence of the urachus often is signaled by a complication. Infection is the most common complication and the most dangerous is his degeneration. The infection can be misunderstood and confused with other pathologies of the navel.

Methods: Our study reports three clinical cases of infected urachal cyst.

Result: these report cases illustrate the need to think of this diagnosis before the painful of the navel and abdomen. The ultrasound examination can help for the diagnosis.
\end{abstract}

Keywords : Urachus, infected cyst, complication

\section{Résumé}

Introduction: L'ouraque est un vestige fibreux de l'allantoïde qui relie le dôme vésical à la paroi abdominale antérieure. Après la naissance elle s'oblitère en général. Dans certains cas, il pourrait persister sous forme de kyste, de fistule, de sinus ou de diverticule. Cette persistance de l'ouraque 
se signale souvent à l'occasion de complications dont l'infection est la plus fréquente et la dégénérescence la plus redoutable. Une telle infection peut être méconnue et confondue à d'autres pathologies de l'ombilic. Méthode: Nous rapportons 3 observations de kyste de l'ouraque infecté. Résultat: ces observations illustrent la nécessité de penser à ce diagnostic devant les symptomatologies douloureuses de l'ombilic et de l'abdomen. Les examens échographiques pourraient être d'une aide pour le diagnostic.

Mots clés : Ouraque, kyste infecté, complication

\section{Introduction}

L'ouraque est un vestige embryonnaire d'origine allantoïdienne et qui se présente à la naissance sous forme d'un cordon fibreux oblitéré, reliant le dôme vésical à l'ombilic. [1,2]. Son rôle n'est pas défini [2]. L'absence d'oblitération de ce vestige peut être à l'origine de malformations diverses qui peuvent rester muettes ou se manifester à l'occasion d'une complication infectieuse ou dégénérative. Nous rapportons trois observations de ce vestige chez des garçons dont la symptomatologie peu spécifique a entrainé des confusions diagnostiques.

\section{Observations cliniques}

\section{Cas 1}

Nourrisson O. âgé de 04 mois, sexe masculin, porteur d'une hernie ombilicale depuis la naissance, a été référé d'un hôpital de zone rural pour hernie ombilicale. La maman a signalé des pleurs incessantes depuis 3 jours, exacerbées à la palpation de l'ombilic sans troubles de transit. Il n'y a pas de notion de fièvre mais le centre référant a noté une fébricule à $37,8^{\circ} \mathrm{C}$. A l'admission on retrouvait un état général conservé, une température à $36,9^{\circ} \mathrm{C}$, une tuméfaction ombilicale globuleuse de $3 \mathrm{~cm}$ de diamètre, non expansive, non réductible et très douloureuse à la palpation. Le reste de l'abdomen est non ballonné, souple, sans masse palpable. Ailleurs, l'examen physique est normal.

Le diagnostic retenu était celui d'une hernie ombilicale étranglée. Une radiographie de l'ASP réalisée par l'hôpital référant montre une distension aérique généralisée des anses sans niveau hydro-aérique. L'échographie, nit et la tomodensitométrie n'étaient pas disponibles dans notre centre hospitalier.

Le patient a été opéré sous anesthésie générale. A travers une incision longitudinale à cheval sur l'ombilic, une dissection du sac herniaire a été réalisée. Ce sac était très épaissi. À son ouverture, on découvre une anse du sigmoïde qui s'est réduite spontanément et qui ne présentait aucune anomalie. L'exploration découvrait un cordon épais inflammatoire, 
s'étendant de l'ombilic au dôme vésical. Ce cordon reconnu comme l'ouraque est recouvert de fausse membrane avec un filet de pus à sa base. On réalise la résection du sac herniaire et de l'ouraque ainsi qu'une cure herniaire par raphie. Un examen anatomo-pathologique n’a pas été possible. Les suites opératoires étaient simples. Le nourrisson était sorti à J12 post opératoire et l'évolution a été favorable après un recul de 4 mois.

\section{Cas 2}

Il s'agissait d'un enfant agé de 5 ans et de sexe masculin, sans antécédent particulier, amené par sa mère pour une augmentation progressive du volume de l'abdomen dans un contexte de pleurs épisodiques avec passage de la main sur l'ombilic évoluant depuis quelques jours. Des tentatives de traitement par la phytothérapie traditionnelle se sont révélées infructueuses.

A l'examen, la température était normale à $37,2^{\circ} \mathrm{C}$, un abdomen augmenté de volume avec ombilic proéminent, déplissé luisant non expansif lors des pleurs, centré par une zone d'abcès. La palpation était douloureuse avec une défense péri-ombilicale. A la percussion, l'abdomen était tympanique dans son ensemble. Les bruits péristaltiques étaient présents. Ailleurs l'examen physique est normal. La numération leucocytaire montrait une hyperleucocytose à 11,770 G/L. Le diagnostic d'omphalite suppurée a été retenu.

Au bloc opératoire à l'incision on notait une poche à membrane épaissie, extra-péritonéale remplie de pus, allant de l'ombilic vers la vessie (figure1). Cette poche n'est rien d'autre qu'un kyste de l'ouraque infecté. Il a été complètement réséqué. Un examen anatomo-pathologique n’a pas été réalisé.

Les suites opératoires ont été simples. Il a été mis en exéat à J15 post opératoire L'évolution ultérieure est sans complication à huit mois de recule.

\section{Cas 3}

Garçon A.K. de trois ans, reçu en urgence pour tuméfaction ombilicale douloureuse évoluant depuis cinq jours avec fièvre. A l'examen, le patient était fébrile à $38^{\circ} 4 \mathrm{C}$, l'ombilic était déplissé, luisant, blanchâtre avec un halo érythémateux d'environ cinq centimètres de diamètre autour de lui (Figure 2). Il était douloureux et fluctuant. La radiographie de l'abdomen sans préparation était normale. Une omphalite suppurée était retenue.

A l'incision, on tombe sur une poche extrapéritonéale remplie de pus dont le prolongement se trouvait en continuité avec l'ouraque épaissi. Elle est réséquée avec le reste de l’ouraque jusqu'en zone saine. Les suites étaient simples. 


\section{Discussion}

L'ouraque est un vestige fibreux de l'allantoïde qui relie le dôme vésical à la paroi abdominale antérieure. Il est extra péritonéal sous facial limité latéralement par les cordons fibreux des artères ombilicales (Richard R. et al., 2011). Entre la cinquième et la douzième semaine de vie intrautérine, ce canal allantoïdien s'étire et s'oblitère donnant le ligament ombilical médian ou l'ouraque (Taki I.Z.2014) Ce vestige de $8 \mathrm{~mm}$ d'épaisseur, est normalement d'une longueur située entre 5 et $10 \mathrm{~cm}$ (Richard R. et al., 2011). La persistance de ce canal allantoïdien à la naissance détermine diverses anomalies congénitales dont l'expression clinique variable peut être latente, muette ou révélée par des complications. Ces anomalies congénitales de l'ouraque sont : le kyste de l'ouraque, une poche présente sur le trajet du ligament sans communication avec la vessie ni l'ombilic dû à l'oblitération en crânial et en caudal ;

la fistule de l'ouraque, communiquant la vessie à l'ombilic, est la persistance du canal allantoïdien sur tout son trajet. Son expression clinique est univoque et précoce : l'écoulement d'urine par l'ombilic ; on parle de fistule ombilico-vésicale ;

le sinus de l'ouraque; formation kystique communiquant avec l'ombilic est le fait de l'oblitération distale de l'ouraque et le défaut d'oblitération en proximal ; c'est le kyste ombilical encore appelé sinus externe ;

quant au diverticule de l'ouraque, structure ouraquienne kystique s'ouvrant dans la vessie, il résulte de l'oblitération crâniale de l'ouraque alors que la partie caudale ne l'est pas. [Arifa $\mathrm{N}$ et al,2004 ; Richard R et al., 2011 ; Taki IZ,2014).

Galati et al. ont observé qu'à la naissance, la persistance du canal ouraquien s'oblitère dans $80 \%$ des cas chez les nourrissons de moins de six mois (Galati V et al.,2008). La prévalence de la pathologie malformative ouraquienne est faible estimée à 0,8 cas par an selon Ndour et al. au Sénégal [Ndour O et al. 2013]. Mais cette prévalence est sans doute sous estimée en raison du fait que la malformation est souvent muette et lorsqu'elle se manifeste à l'âge adulte c'est souvent par une dégénérescence maligne (Ashley RA et al.,2007 ; Tian J et al. 2008 ;Thali-Schwab et al.,2005 ; Ashley RA. et al.,2006). La fréquence d'une forme d'anomalie varie d'une étude à une autre [Ndour O et al., 2013 ; Ashley RA et al.,2007).

Les anomalies de l'ouraque sont plus fréquentes chez le sujet de sexe masculin (Ndour O et al, 2013 ; Ashley RA et al, 2007;Josef G, 2014) nos observations concernaient des garçons. Les patients porteurs d'anomalies de l'ouraque sont généralement asymptomatiques, en dehors de la fistule ombilico-vésicale qui s'exprime très tôt par l'écoulement urinaire à l'ombilic en période néonatale. 
Comme le montrent nos cas, les manifestations cliniques des pathologies de l'ouraque, en dehors de la fistule ombilico-vésicale, sont des complications dont les signes n'ont rien de spécifique: la suppuration ombilicale, la douleur, un érythème ou une masse ombilicale simulant un étranglement herniaire. La douleur abdominale peut être le seul symptôme de la maladie. Cette douleur peut simuler un abdomen aigu (Qureshi K et al, 2013). Le diagnostic différentiel se fait devant une masse ombilicale avec un hématome, un abcès, une hernie ombilicale, et une tumeur de la paroi abdominale. L'échographie peut être utile mais elle n'est pas suffisante, elle ne permet de mettre en évidence l'ouraque normal que dans $62 \%$ des cas (Arifa $\mathrm{N}$ et al, 2004). Elle n'est pas de routine dans notre contexte où les spécialistes d'imagerie sont en sous effectif et les malades sans moyens pour faire face aux dépenses de soins de façon convenable. La TDM est l'examen le plus indiqué, mais la meilleure précision est donnée par l'IRM (Arifa $\mathrm{N}$ et al,2004;Nimmonrat A et al,2008). Ces examens d'imagerie sont encore des luxes dans nos contextes et ne peuvent être demandés à tout venant. L'équipement de tomodensitométrie le plus proche est à soixante kilomètres du centre tandis qu'il n'existe encore aucun appareil d'IRM dans le pays tout entier.

Dans ces conditions, nos cas ont été diagnostiqués en per opératoire étant donné le caractère aigü de la symptomatologie qui faisait le change avec un étranglement herniaire ou une omphalite. La rareté de l'affection surtout dans un service non spécialisé explique le fait qu'on n’y pense pas en première intention.

.Quand la pathologie de l'ouraque se manifeste par des signes d'infection, une bithérapie s'impose, fait d'une antibiothérapie et de la lutte contre l'inflammation (Yoo KH et al,2006), suivie par une ablation chirurgicale. Nos trois patients avaient bénéficié du traitement approprié. En effet, l'excision complète de l'ouraque avec ou sans l'excision du dôme vésical serait suffisante malgré un risque possible de complications post opératoires [Naiditch JA et al,2013]. Il n'est pas nécessaire d'enlever l'ombilic. En cas de cancer de l'ouraque, on envisagera une cystectomie partielle ou radicale. La cystectomie partielle avec la résection en bloc de l'ouraque et du dôme de la vessie donne des résultats semblables à la cystectomie radicale au plan oncologique [Henly DR et al,1993].

\section{Conclusion}

La pathologie de l'ouraque est rarement évoquée dans la pratique quotidienne dans notre contexte. Pourtant, comme le montrent ces trois cas rencontrés en quelques mois d'intervalle, elle n'est pas si rare. Devant les cas de hernie ombilicale étranglée de l'enfant, d'infections ombilicales il faut y penser et demander l'échographie. 


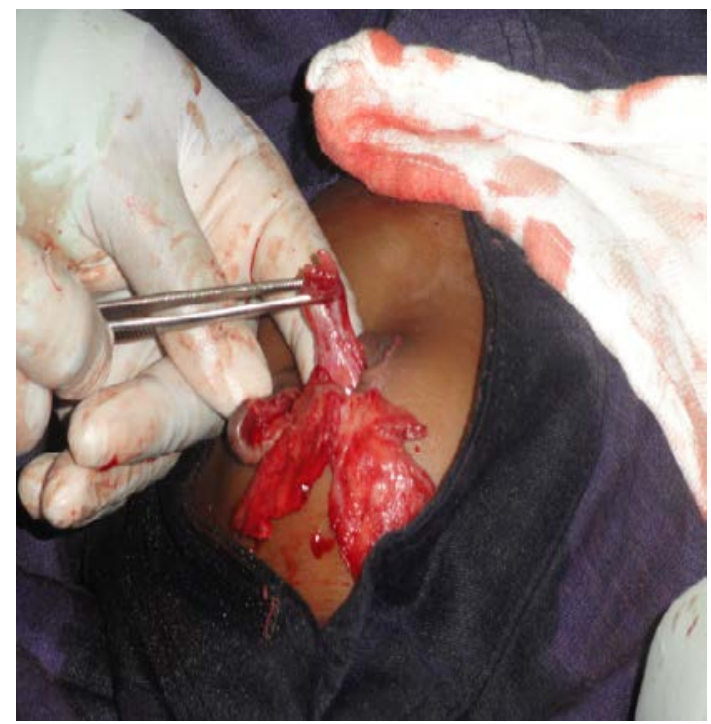

Figure 1 : Kyste de l'ouraque infecté : dissection

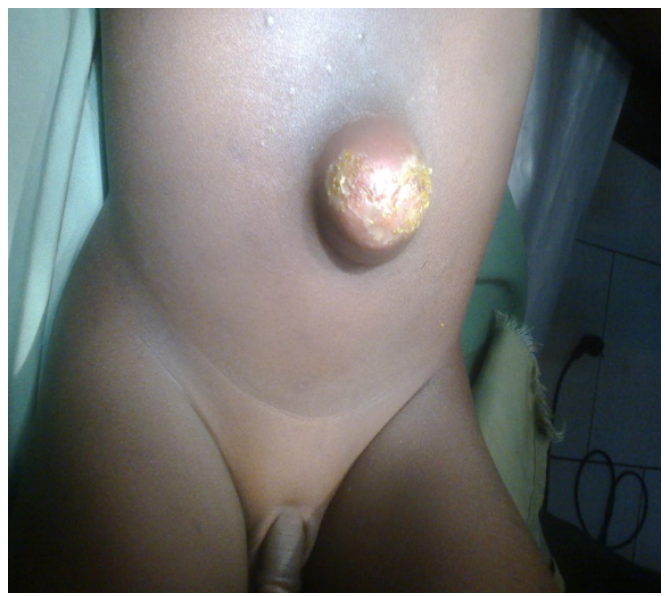

Figure 2 : Ombilic déplissé, luisant, blanchâtre, halo érythémateux

\section{References:}

Arifa N, Hasni I, Khadraoui H , Mhiri M, Jemni H, Derbel F, Ben Sorba N, Tlili Graiess $\mathrm{K}$.Atlas d'imagerie de l'ouraque du normal au pathologique. JFR2004

Ashley RA, Inman BA, Routh JC, Rohlinger AL, Husmann DA, Kramer SA.2007. Urachal anomalies: a longitudinal study of urachal remnants in children and adults. J Urol.;178:1615-1618. [PubMed].

Ashley RA, Inman BA, Sebo TJ, Leibovich BC, Blute ML, Kwon ED, Zincke H. 2006. Urachal carcinoma: clinicopathologic features and long- 
term outcomes of an aggressive malignancy. Cancer.;107:712-720. [PubMed

Galati V, Donovan B, Ramji F, Campbell J, Kropp BP, Frimberger D. 2008. Management of urachal remnants in early childhood. J Urol. (suppl 4):18241826. [PubMed].

Henly DR, Farrow GM, Zincke H. 1993. Urachal cancer: role of conservative surgery. Urology.;42:635-639. [PubMed].

Josef G. le kyste de l'ouraque chez l'adulte, à propos de 9 cas. Etude rétrospective. www.rdgn.ucl.ac.be/fr/Staff/mars_18\%20.pdf consulté le 30 / 4/ 2014.

Naiditch JA, Radhakrishnan J, Chin AC. 2013. Current diagnosis and management of urachal remnants, J Pediatr Surg. Oct;48(10):2148-2152.

Ndour O, Mukulu HK , Ndoye NA , Mbaye PA , Faye Fall A, Ngom G , Ndoye M. 2013.La pathologie malformative de l'ouraque: A propos de 12 cas colligés au service de Chirurgie Pédiatrique du CHU Aristide Le Dantec de Dakar, Sénégal. Afr Jour of urology, 19 :115-118

Nimmonrat A, Na-Chiang Mai W, Muttarak M. 2008. Urachal abnormalities: clinical and imaging features. Singapore Med J.;49:930-935. [PubMed].

Qureshi K, Maskell D, McMillan C, Wijewardena C. 2013. An infected urachal cyst presenting as an acute abdomen - A case report. Int J Surg Case Rep.;4:633-635. [PubMed]..

Richard R, Bouanane M, Salomon L, Vordos D, Pigneur F, Werbrouck A, Rahmouni A, Luchani A. 2011. Pathologie de l'ouraque : kyste de l’ouraque infecté à propos d'un cas. Journal de radiologie ; 92 : 250-253.

Taki Imrani Zineb ; 2014. Les abcès de l'ouraque chez l'adulte, à propos de cinq cas et revue de littérature. Thèse de Doctorat en Médecine ; Faculté de Médecine et de Pharmacie de FES, Université SIDI MOHAMMED BEN ABDELLAH. FES ; n¹35/14 : p144.

Thali-Schwab CM, Woodward PJ, Wagner BJ. 2005.Computed tomographic appearance of urachal adenocarcinomas: review of 25 cases. Eur Radiol. 15:79-84. [PubMed].

Tian J, Ma JH, Li CL, Xiao ZD. 2008.Urachal mass in adults: clinical analysis of 33 cases. Zhonghua Yi Xue Za Zhi.;88:820-822. [PubMed].

Yoo KH, Lee SJ, Chang SG. 2006. Treatment of infected urachal cysts. Yonsei Med J.;47:423-427. [PMC free article] [PubMed]. 